\title{
Sur le genre Citellina Prendel, 1928 (Oxyuridae, Nematoda)
}

\author{
par J.-P. HUGOT
}

Laboratoire de Zoologie (Vers) associé au C.N.R.S., Muséum national d'Histoire naturelle, 43, rue Cuvier, 75231 Paris Cedex 05.

\section{Résumé.}

Cinq des treize espèces du genre Citellina Prendel, 1928, sont reconnues valides d'après leurs structures céphaliques. Citellina sp. Quentin, 1968, devient C. caligata n. sp.; C. alatau Spassky, Rijikov et Sudarikov, 1950, est réexaminée et déclarée valide. Une quatorzième espèce est décrite : Citellina undulata n. sp., de Citellus undulatus en Alaska. C. petrovi Schulz, 1930 est redécrite.

Les affinités et les caractéristiques évolutives du genre Citellina sont étudiées. Cinq types céphaliques peuvent y être distingués et chacun correspond à un Sciuridé particulier. Les parasites de Citellus et de Marmota forment un groupe distinct et leurs morphologies céphaliques respectives reproduisent le dimorphisme sexuel observé dans certaines espèces du genre Auchenacantha Baylis, 1929. La distribution géographique du genre Citellina coïncide avec celle des Citellus. Les genres Auchenacantha et Citellina, quoique proches, paraissent s'être séparés depuis longtemps. L'origine du genre Citellina semble pouvoir être situé dans le centre-est Asiatique, d'où il se serait dispersé et différencié en même temps que les Ecureuils holarctiques.

\section{Sụminary.}

\section{On the genus Citellina Prendel, 1928 (Oxyuridae, Nematoda).}

The cephalic structures were described for five species among the thirteen ones from the genus Citellina Prendel, 1928 ; these characters allow to valid the following species : $C$. triradiata (Hall, 1916) ; C. marmotae Manter, 1930 ; C. petrovi Schulz, 1930 ; C. levini Li, 1933 ; C. himalensis Inglis \& Ogden, 1965.

Three species are re-examinated : Citellina sp. Quentin, 1968 becomes C. caligata n. sp.; C. alatau Spassky, Rijikov and Sudarikov, 1950 is validated; C. petrovi is redescribed. A fourteenth species is described : C. undulata n. sp. from Citellus undulatus in Alaska. Five cephalic types are distinguished, every one is associated with a peculiar host : Sciurus, Citellus, Marmota, Pteromys and Callosciurus. The deep-rooted affinities between the genera Citellina and Auchenacantha Baylis, 1929, are confirmed. The Citellinae from Citellus and

Accepté le 9 novembre 1979. 
Marmota are isolated into separate group because of their peculiar cephalic morphologies which display the same dimorphism observed in some Auchenacantha species, and because of the special shape of their eggs. All the Citellina were described from Sciuridae from the holarctic region and the geographical distribution of this genus coincides with the distribution of the genus Citellus. The development and differentiation of the Citellina apparently fit with those of the holarctic squirrels.

\section{Introduction}

Depuis la création du genre Citellina, 13 espèces y ont été décrites. Certaines doivent probablement être mises en synonymie, mais, dans 5 des cas, les structures céphaliques sont connues et permettent de déclarer les espèces certainement valides. Il s'agit de C. triradiata (Hall, 1916), C. marmotae Manter, 1930, C. petrovi Schulz, 1930, C. levini Li, 1933, C. himalensis Inglis et Ogden, 1965.

Trois des espèces connues: C. petrovi Schulz, 1930, C. alatau Spassky, Rijikov et Sudarikov, 1950 et Citellina sp., Quentin, 1971 sont réétudiées dans la partie morphologique.

Six autres espèces : C. dispar Prendel, 1928, C. schulzi Korneev, 1951, C. alpina Jettmar et Anschau, 1951, C. skjrabini Merkusheva, 1955, C. bashiri Akhtar, 1956 et, C. hindukushensis Erhardova-Kotrla et Daniel, 1970, n'ont pas été étudiées en vue apicale et ne peuvent donc être utilisées pour tenter d'interpréter l'évolution du genre. En outre, aucune d'elles ne peut être rapprochée des espèces nouvelles que nous avons à décrire: nous ne les prendrons donc pas en considération.

\section{Morphologie}

Le matériel utilisé a été transmis par le $\mathrm{D}^{r}$ Rausch, auquel nous adressons nos remerciements les plus vifs. Tous les spécimens sont déposés au Muséum national d'Histoire naturelle.

\section{Citellina undalata $\mathrm{n} . \mathrm{sp}$.}

\section{MATÉRIEL :}

54 mûres, récoltées chez un Citellus undulatus $q$, capturé le 21-VIII-69 à Inukpasukruk Creek (east of Anaktuvuk Pass), central Brooks Range (Alaska). MNHN : $731 \mathrm{M}$.

\section{DESCRIPTION :}

Ouverture buccale en forme de triangle à base dorsale, entourée par trois lèvres, découvrant trois dents œsophagiennes coniques, peu développées. Pharynx renforcé 
à sa périphérie par un anneau chitineux. Cycle interne des papilles labiales visible. Papilles céphaliques disposées en carré : deux sur la lèvre dorsale, une sur chacune des lèvres latéro-ventrales. Ecart des pores amphidiaux chez un spécimen : $41 \mu \mathrm{m}$ (fig. 1, A). Eufs non embryonnés, portant deux longs filaments polaires (fig. 2, G). Lèvres vulvaires antérieure et postérieure également renflées (fig. 2, C). Au niveau de l'anneau nerveux naissent deux ailes latérales, renforcées (fig. 2, F), qui se prolongent jusqu'à l'anus. Moyenne des mensurations de cinq spécimens, en millimètres :

Longueur du corps : 4,65; largeur du corps: 0,395; longueur de la vésicule céphalique : 0,137 ; longueur de l'œsophage total : 0,372 ; longueur du bulbe : 0,084 ; largeur du bulbe : 0,116; distance apex-anneau nerveux : 0,185; apex-pore excréteur : 0,185 ; apex-vulve : 1,128 ; queue : 0,84 ; moyenne des mensurations de 10 œufs: $0,075 \times 0,036$.

\section{Discussion :}

Deux autres espèces présentent une bouche entourée par trois lèvres bien développées (fig. 1) : - chez C. levini, les mensurations sont très différentes et les œufs sont d'un autre type, puisqu'ils ne portent pas de filaments ; $-C$. triradiata présente des mensurations très proches et des œufs de même type. Hall ne donne pas de vue apicale mais décrit trois lèvres bien développées et donne une coupe optique de la tête en vue ventrale très semblable à celle que nous observons (fig. $1, B$ et $1, C$ ). Nos animaux nous paraissent pourtant différents pour les caractères suivants : œsophage plus court, vulve située plus antérieurement, présence d'ailes latérales. Les parasites de $C$. undulatus appartiennent donc à une espèce nouvelle: Citellina undulata $\mathrm{n}$. sp. du nom de son hôte.

\section{Citellina ealigata n. sp. = Citellina sp. Quentin, 1968}

MATÉRIEL :

C’est celui déjà décrit par Quentin chez Marmota caligata. MNHN : 66 h.

\section{Discussion :}

Dans sa conclusion, Quentin pensait, sans en avoir la certitude, pouvoir identifier son espèce à $C$. triradiata. Il convient plutôt de comparer le matériel de Quentin à $C$. marmotae, qui est la seule espèce à présenter également 6 lobes labiaux (fig. $1, D$ et $1, F)$.

Les mensurations de $C$. marmotae et Citellina sp. sont très proches. On peut toutefois les distinguer pour les caractères suivants :

- C. marmotae: une seule dent sur chacun des lobes labiaux; pharynx court et inerme ; 2 renflements vulvaires ; gubernaculum peu visible, long (mesuré sur le dessin de Read, p. 449, fig. 1, plate 1) de $20 \mu \mathrm{m}$; écart des pores amphidiaux : $120 \mu \mathrm{m}$. 

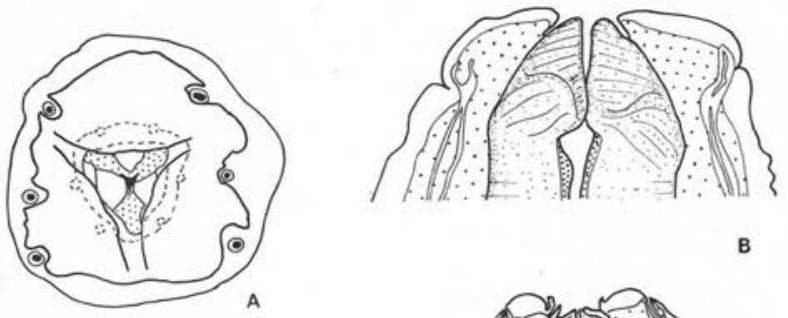

B
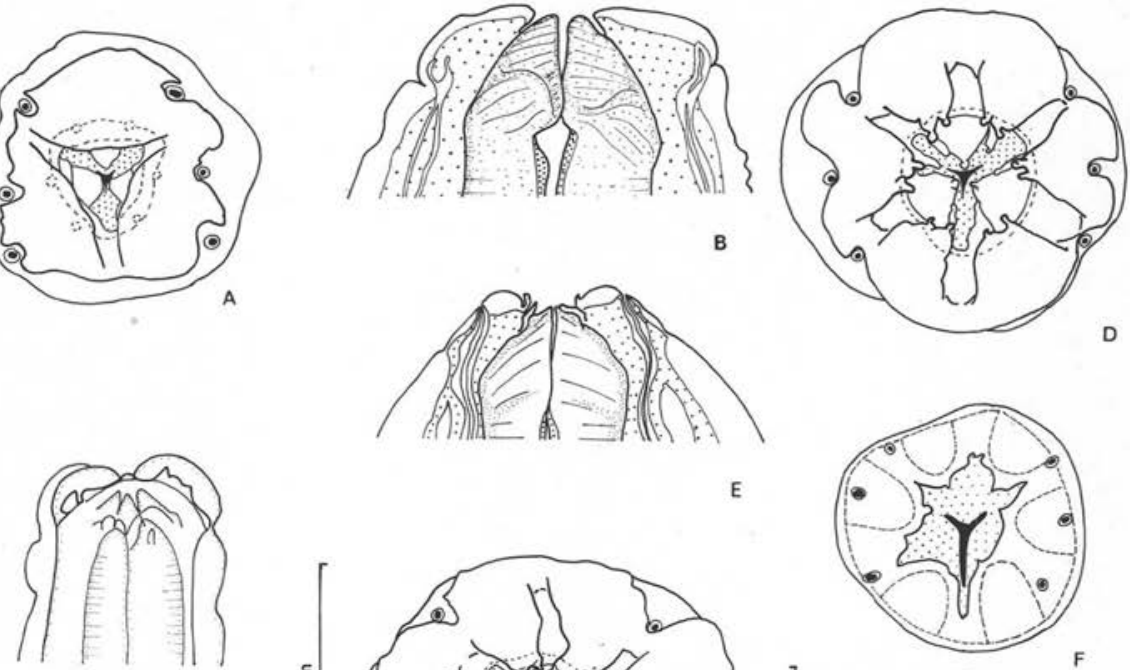

D
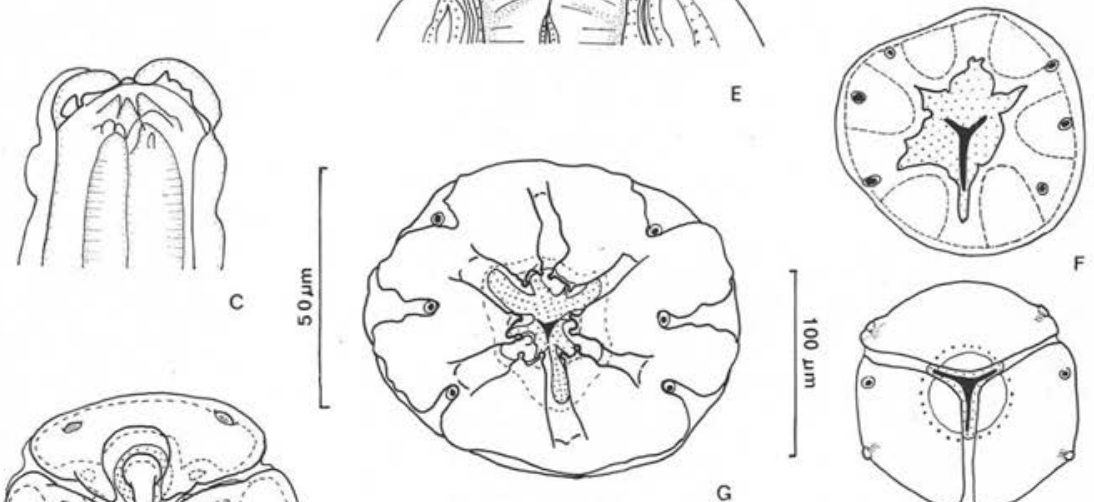

c

E
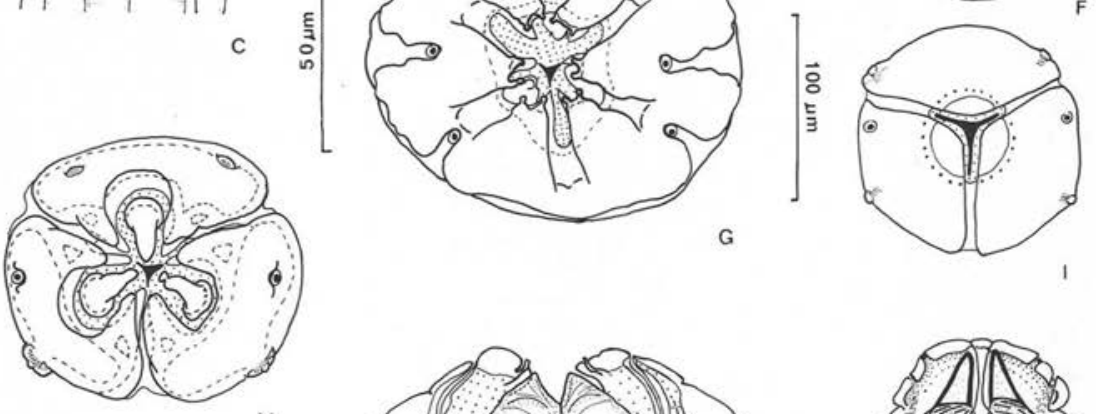

M
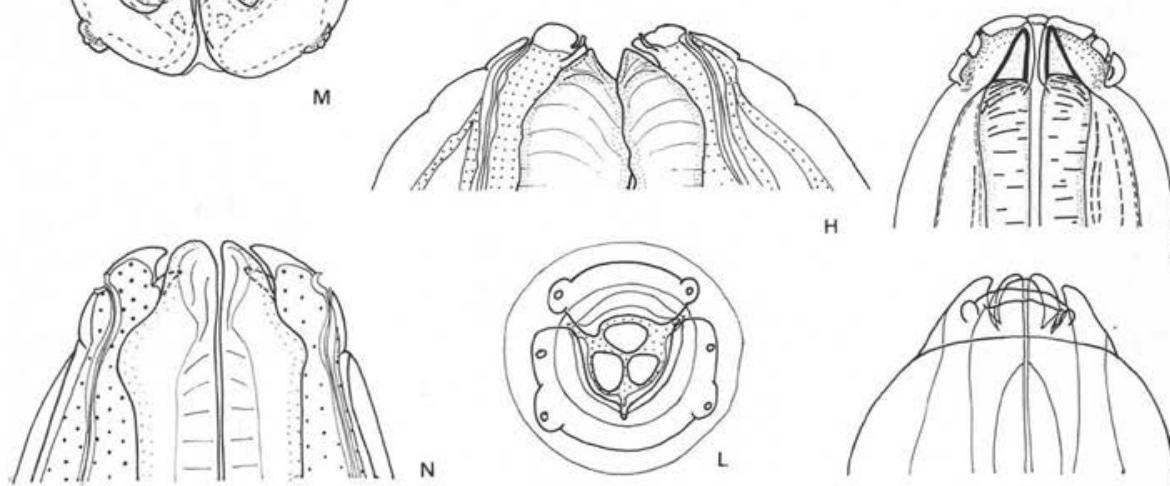

$\mathrm{H}$

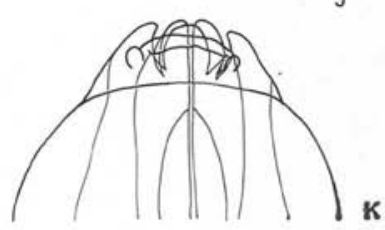

Fig. 1. C. undulata $९$ n. sp.: A, tête, vue apicale ; B, id., coupe optique, vue ventrale ; $-C$. triradiata : C, tête, vue ventrale (d'après Hall) ; $-C$. caligata ? n. sp.; D, tête, vue apicale ; $\mathrm{E}$, id., coupe optique, vue ventrale ; - C. marmotae : F, tête, vue apicale (d'après Manter) ; C. alatau ? : $\mathrm{G}$, tête, vue apicale; $\mathrm{H}$, id., coupe optique, vue ventrale ; - C. levini : $\mathrm{I}$, tête, vue apicale ; J, id., coupe optique, vue ventrale (d'après $\mathrm{Li}) ;-C$. himalensis : $\mathrm{L}$, tête, vue apicale ; $\mathrm{K}$, id., vue ventrale (d'après Inglis et Ogden) ; $-C$. petrovi : $\mathrm{M}$, tête, vue apicale ; $\mathrm{N}$, id., vue ventrale.

A, B, D, E, G, H, I et J, éch. : $50 \mu \mathrm{m}$; F, éch. : $100 \mu \mathrm{m}$; C, K, L, M et N, pas d'échelle. 
- Parasites de M. caligata : trois dents sur chaque lobe labial ; pharynx occupé par trois dents œsophagiennes hautes et portant chacune un denticule (fig. 1, D); un seul renflement vulvaire (postérieur) (fig. 2, A); gubernaculum bien visible, long de $42-45 \mu \mathrm{m}$; écart des pores amphidiaux : $55 \mu \mathrm{m}$.

Les animaux décrits par Quentin font donc partie d'une espèce nouvelle : Citellina caligata $\mathrm{n}$. sp., du nom de leur hôte.

\section{Citellina alatau Spassky, Rijikov et Sudarikov, 1950}

\section{MAtÉRIEL :}

Nombreux $\delta^{*}$ et $q$ récoltés chez Marmota menzbieri, le 30-VII-61, à l'Ouest du Tyan-Shan (Kirghizie), à une altitude de $2200 \mathrm{~m}$. MNHN : $612 \mathrm{~S}$.

\section{COMPLÉMENTS MORPHOLOGIQUES :}

Dans les deux sexes, ouverture buccale en forme d'étoile, découpée par 6 lobes labiaux. Chaque lobe se termine par deux denticules égaux et parallèles, et, plus profondément, par une dent labiale; 3 dents œsophagiennes de petite taille occupent l'arrière-cavité buccale (fig. $1, G$ ). Deux ailes latérales renforcées naissent au niveau de l'anneau nerveux et se prolongent jusqu'à l'anus ou au cloaque (fig. $4, I$ ). Chez la 9 , les lèvres vulvaires forment deux renflements de même taille (fig. 2, B).

Mensurations en mm d'un mâle: écart des pores amphidiaux : 0,047; longueur du corps : 6,625 ; largeur du corps : 0,26 ; longueur de la vésicule céphalique : 0,21 ; distance apex-anneau nerveux : 0,225 ; apex-pore excréteur : 1,84 ; œsophage total : 0,62 , dont un bulbe de $0,125 \times 0,130$; spicule : 0,13 ; gubernaculum : $0,035 \times 0,020$; queue : 0,165 .

Mensurations en mm d'une femelle : écart des pores amphidiaux : $0,048(0,053 \mathrm{chez}$ un autre spécimen) ; longueur du corps : 10,725; largeur : 0,575; longueur vésicule céphalique : 0,25 ; distance apex-anneau nerveux : 0,28 ; apex-pore excréteur: 1,8 ; apex-vulve : 2,2 ; œsophage total : 0,7 , dont un bulbe de $0,15 \times 0,15$; queue : 1,7 ; dimensions moyennes de 10 œufs : $0,070 \times 0,040$.

\section{Discussion :}

Les mensurations de ces animaux, en particulier celles des organes copulateurs et des œufs, sont très proches de celles de $C$. alatau, ils proviennent du même hôte et de la même région géographique : on peut les identifier à l'espèce décrite par les auteurs russes, et dont la validité a été contestée par Read (1957), puis Inglis et Ogden (1965). Il est donc nécessaire de la comparer aux deux autres espèces montrant une bouches à 6 lobes labiaux: $C$. marmotae et $C$. caligata n. sp. 

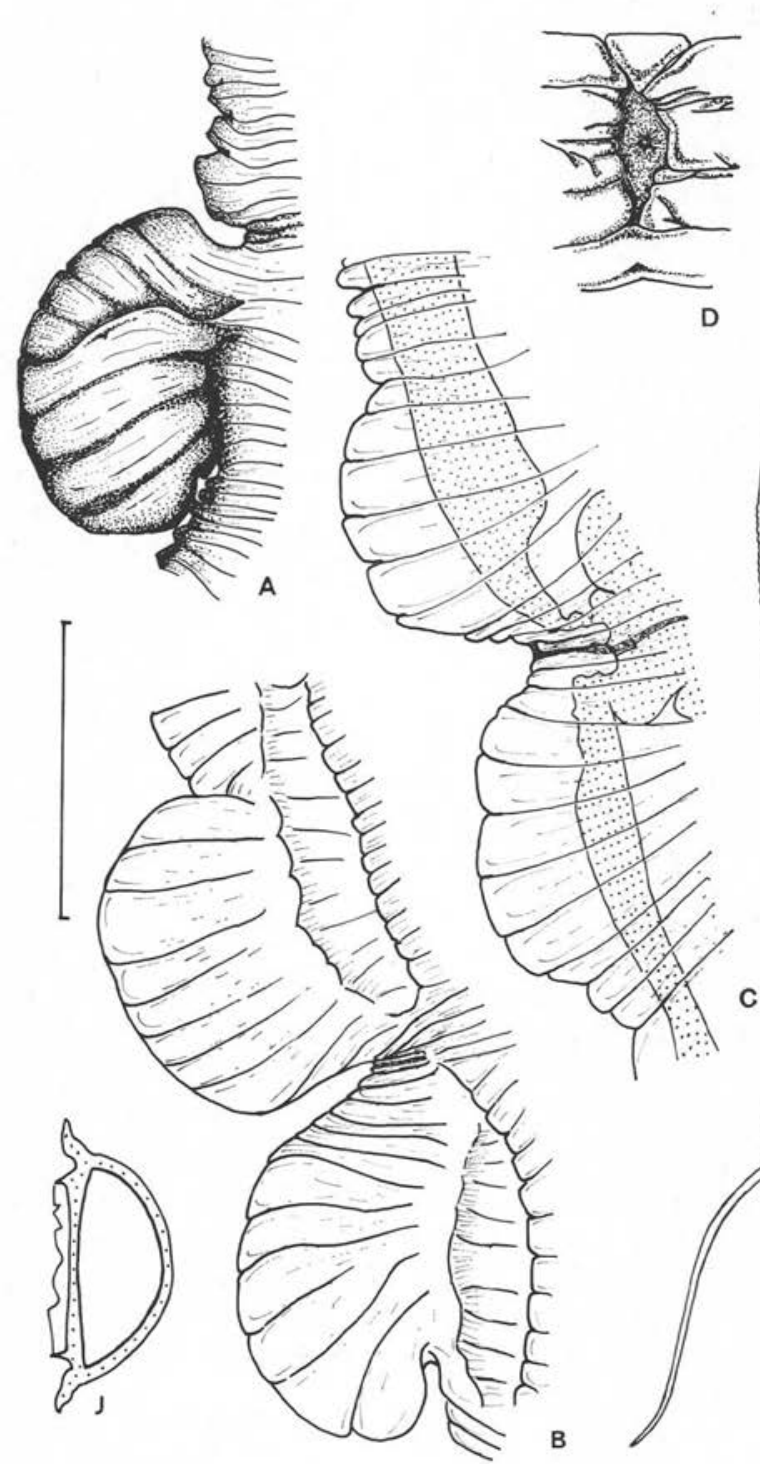

A
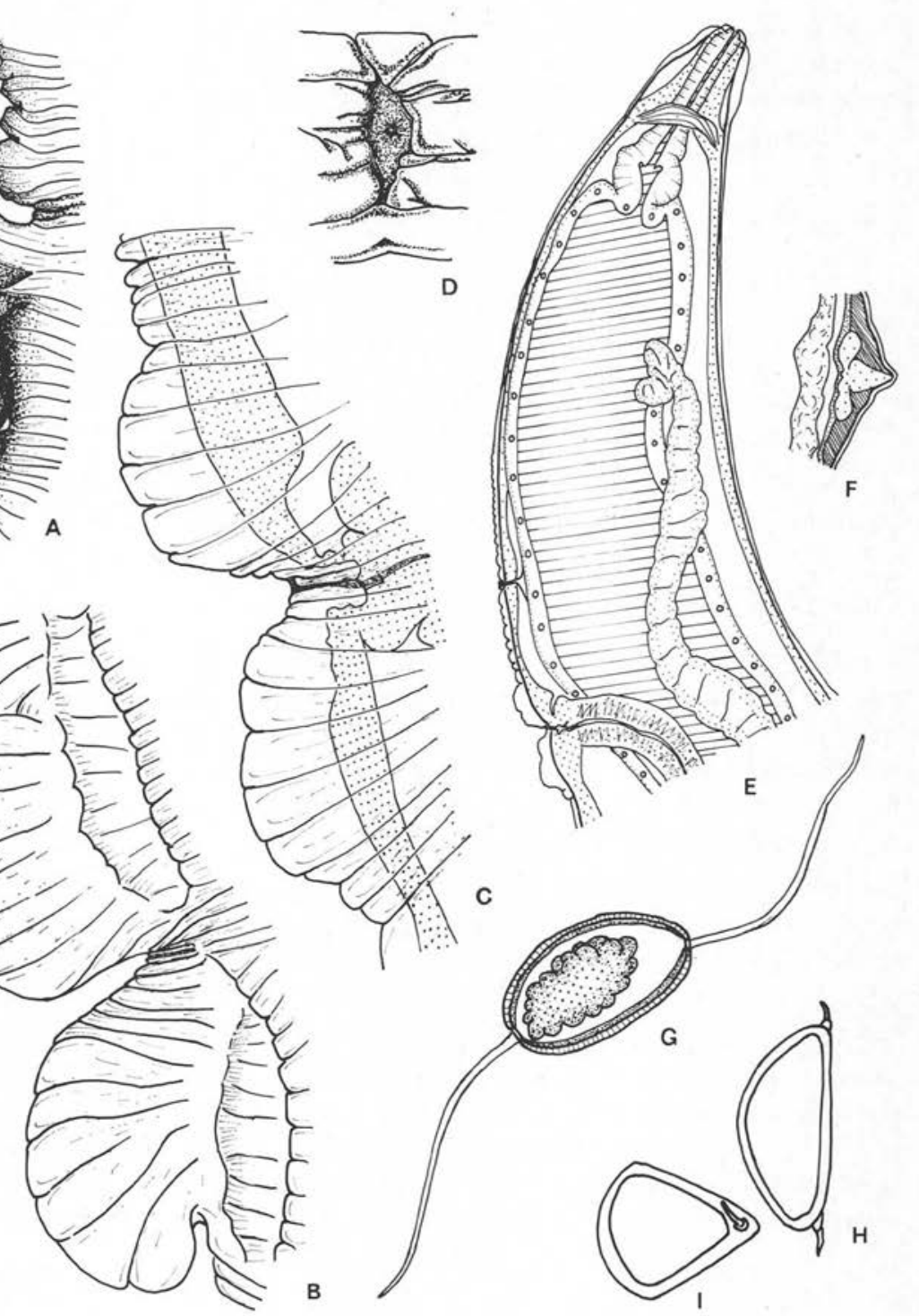

Fig. 2. C. caligata : A, vulve, vue latérale gauche; - C. alatau: B, vulve, vue latérale gauche ; - C. undulata n.sp. : C, vulve, vue latérale gauche; D, pore excréteur, vue ventrale; E, extrémité antérieure, vue latérale gauche ; F, aile latérale, coupe transversale ; $\mathrm{G}$, œuf ; $-C$. himalensis : $\mathrm{H}$, œuf ; I, id., coupe optique (d'après Inglis et Ogden) ; $-C$. petrovi : J, œuf (d'après Schulz).

Valeur de l'échelle suivant les figures: A, B : $250 \mu \mathrm{m}$; C, D, F : $50 \mu \mathrm{m}$; E : $500 \mu \mathrm{m}$; $\mathrm{G}: 100 \mu \mathrm{m} ; \mathrm{H}, \mathrm{I}, \mathrm{J}$, pas d'échelle. 
Les parasites de $M$. menzbieri se différencient de $C$. marmotae par la présence sur chaque lobe labial de deux denticules et d'une dent, au lieu d'une seule dent; par l'existence de deux renflements vulvaires égaux; par un spicule beaucoup plus court. Ils se différencient de $C$. caligata n. sp. par des dents œsophagiennes moins développées, par l'existence de deux renflements vulvaires et par un spicule beaucoup plus court. $C$. alatau est donc nettement distincte des espèces qui lui sont les plus proches, et peut être considérée comme valide.

\section{Citellina petrovi Schulz, 1930}

MATÉRIEL :

9 et $4 \delta^{\star}$ adultes récoltés chez Pteromys volans sur la rivière Kolyma (Sibérie) par $62^{\circ}$ IN le 5-8-79. MNHN : $182 \mathrm{MB}$.

Dans les deux sexes : terminaisons nerveuses portées par trois fortes lèvres débordant largement le plateau céphalique et découvrant les dents œsophagiennes. Chaque lèvre se divise en deux lobes latéraux, eux-mêmes prolongés par une dent labiale plus profonde. Les dents labiales encadrent deux à deux la dent œsophagienne correspondante (fig. 1, $M$ et $N$ ). Les lèvres sont séparées du corps par un sillon cuticulaire profond. Vésicule céphalique peu développée. Deux ailes cervicales naissent immédiatement en arrière de la tête et se prolongent par des ailes latérales larges. Chez la $\nmid$, les ailes se terminent peu avant l'anus; chez le mâle, elles se terminent dans la bourse caudale (fig. 3, D).

Mensurations eN mM D'Un MÂle : écart des pores amphidiaux : 0,04; longueur du corps : 4 ; largeur du corps : 0,22; distance apex-anneau nerveux : 0,14; apex-pore excréteur : 0,58 ; œsophage total : 0,43 ; bulbe : 0,115 ; spicule : 0,175 ; gubernaculum : $0,025 \times 0,018$; queue : 0,155 ; largeur de l'aile latérale au milieu du corps : 0,025 ; boucle testiculaire située à 0,87 de l'apex.

Bourse caudale figurée en : $3, G$.

Mensurations EN MM D'UnE $q$ : écart des pores amphidiaux : 0,055; longueur du corps : 7,75 ; longueur de la vésicule céphalique : 0,28 ; distance apex-anneau nerveux : 0,24 ; apex-pore excréteur : 0,95 ; apex-vulve : 1,45 ; œsophage total : 0,58 ; bulbe : 0,14 ; queue : 2,2 ; œuf : $0,085 \times 0,04$, plus deux prolongements polaires longs de 0,012 chacun; largeur de l'aile latérale au milieu du corps : 0,05.

Chez la $\uparrow$, les stries cuticulaires transversales écartées d'environ $10 \mu \mathrm{m}$ dans la partie antérieure du corps s'espacent progressivement vers l'arrière. L'écart atteint $20 \mu \mathrm{m}$ au niveau de la vulve et $60 \mu \mathrm{m}$ dans le dernier tiers du corps. Il existe une ornementation cuticulaire particulière, sous forme de petites écailles de forme oblongue, disposées régulièrement à la surface des stries transversales ; cette ornementation naît en arrière de l'anneau nerveux et se prolonge jusqu'entre le pore excréteur et la vulve (fig. 3, $A$ et $B$ ). La vulve est entourée par deux renflements peu marqués 


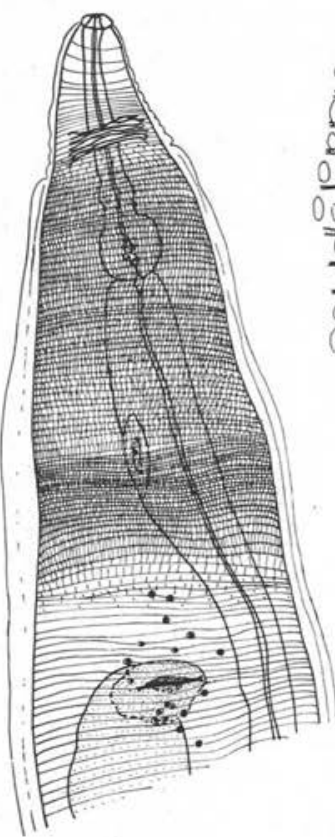

A

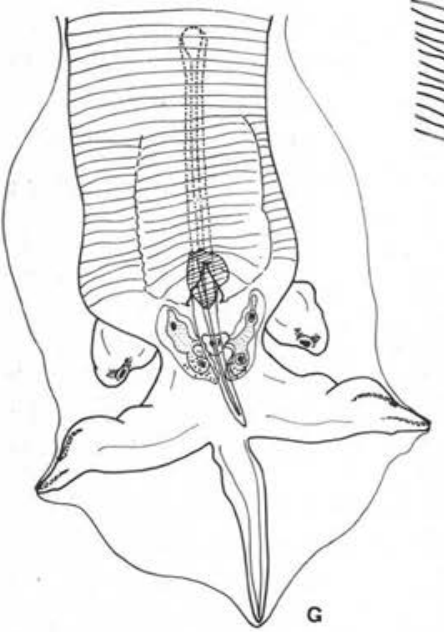

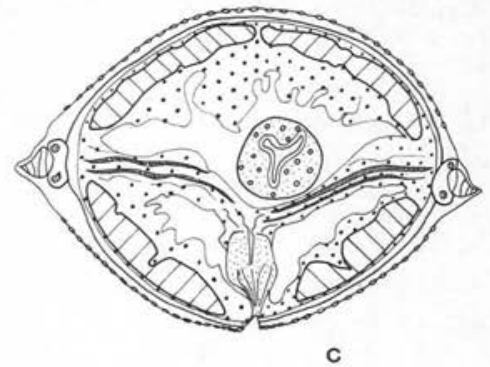
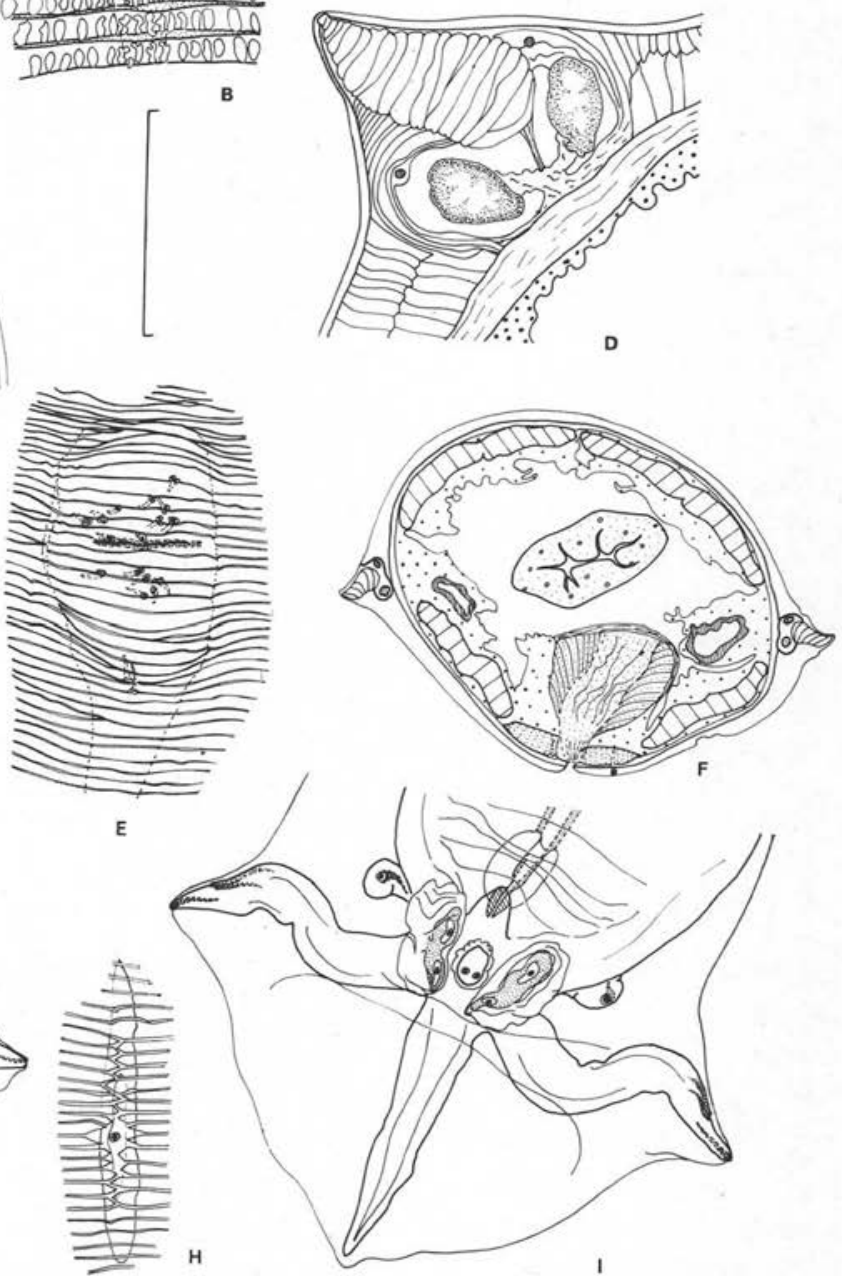

Fig. 3. Citellina petrovi Schulz, 1930. $\&$ : A, partie antérieure du corps, vue ventrale; B, détail de la cuticule dans la région du pore excréteur; C, coupe transversale du corps au niveau du pore excréteur ; D, aile latérale, détail sur une coupe transversale ; E, vulve, détail ; $\mathrm{F}$, coupe transversale du corps au niveau de la vulve. - $\hat{o}: \mathrm{G}$, bourse caudale; $\mathrm{H}$, pore excréteur, détail. - Citellina alatau Spassky, Rijikov et Sudarikov, 1950. ô I, bourse caudale.

Valeur de l'échelle : A, $500 \mu \mathrm{m}$; B et D, $50 \mu \mathrm{m}$; C, E, F, $250 \mu \mathrm{m}$; G, H, I, $100 \mu \mathrm{m}$. 
(fig. $3, E$ ) et porte des formations dont la nature (nerveuse, glandulaire ou parast taire ?) ne peut être précisée sans recours à l'histologie. Des formations analogues ont été décrites par Schulz chez $C$. petrovi et par Prendel et Korneev respectivement chez $C$. dispar et $C$. schulzi.

\section{Discussion :}

Les mensurations et les caractères morphologiques de ces animaux correspondent à ceux décrits par Schulz chez le même hôte. Nous les identifions donc à $C$. petrovi.

\section{Conclusion}

Le genre Citellina n'est connu que chez les Ecureuils et exclusivement dans la zone holarctique. Parmi les huit espèces que nous reconnaissons comme certainement valides, on peut distinguer cinq types céphaliques différents, chacun semblant correspondre à un Sciuridé particulier.

- Type A : trois lèvres distinctes, recouvrant l'ouverture buccale et débordant le plateau céphalique. Les papilles céphaliques et les amphides sont portées par les lèvres (fig. $1, I$ et $J$ ). Ce type est rencontré chez $C$. levini, parasite de Sciurus vulgaris Linné, à Pékin, en Chine ( $\mathrm{Li}, 1933)$. Il rappelle des types faciaux rencontrés chez certains Cosmocercoïdés et Pharyngodonidés, et peut donc être considéré comme primitif.

- Type B : tête ayant la même forme générale et la même disposition des terminaisons nerveuses que le type $\mathrm{A}$, mais les lèvres régressent en découvrant les dents œsophagiennes, chacune de ces dents est encadrée par deux lobes labiaux surmontant chacun une dent labiale plus profonde (fig. 1, M et $N$ ). Ce type est observé chez C. petrovi, parasite de Pteromys volans volans Linné, en Russie d'Europe (Schulz, 1930), P. volans orii Kuroda, au Japon (Kamiya, Ishigari et Yamashita, 1974) et $P$. volans Linné, en Sibérie (voir plus haut). Ce type paraît pouvoir dériver directement du type A.

- Type $C$ : tête ayant la même forme générale et la même disposition des terminaisons nerveuses que les types $\mathrm{A}$ et $\mathrm{B}$. Les lèvres, plus réduites que dans le type $\mathrm{B}$, tendent à confluer (latéro-ventrales partiellement soudées) et former un bourrelet annulaire péribuccal (fig. $1, K$ et $L$ ). Ce type est observé chez $C$. himalensis, parasite de Callosciurus macclellandi macclellandi Horsfield, dans l'Himalaya, au Népal (Inglis et Ogden, 1965). Ce type paraît également dériver directement du type A.

- Type D: trois lèvres soudées à leurs commissures, découvrant plus ou moins les dents œsophagiennes et ne recouvrant pas le plateau céphalique, qui porte les terminaisons nerveuses (fig. 1, $A$ et $B$ ). Ce type est observé chez $C$. triradiata, parasite de Ammospermophilus leucurus cinnamomeus (Merriam) et Citellus (Callospermophilus) lateralis (Say) dans l'Etat du Colorado (Hall, 1916) et chez C. undulata, parasite de C. undulatus Pallas en Alaska (voir plus haut). 
- Type E : tête présentant la même disposition des lèvres et des terminaisons nerveuses que le type $\mathrm{D}$, mais chaque lèvre est subdivisée en 2 lobes égaux, qui se terminent chacun par une dent labiale. Les six dents labiales encadrent 2 à 2 la dent œsophagienne correspondante (fig. 1, D, F, G). Ce type est observé chez $C$. marmotae, parasite de Marmota monax canadensis (Erxleben) dans l'Etat du Maine (Manter, 1930), chez C. caligata, parasite de $M$. caligata (Eschs.) en Alaska (Quentin, 1968) et chez $C$, alatau, parasite de M. marmota menzbieri Kashkarov en Kirghizie (Spassky, Rijikov et Sudarikov, 1950).

Le type céphalique, la morphologie des œufs, la répartition géographique et le spectre d'hôtes permettent de distribuer les Citellina en deux groupes (fig. 4) :

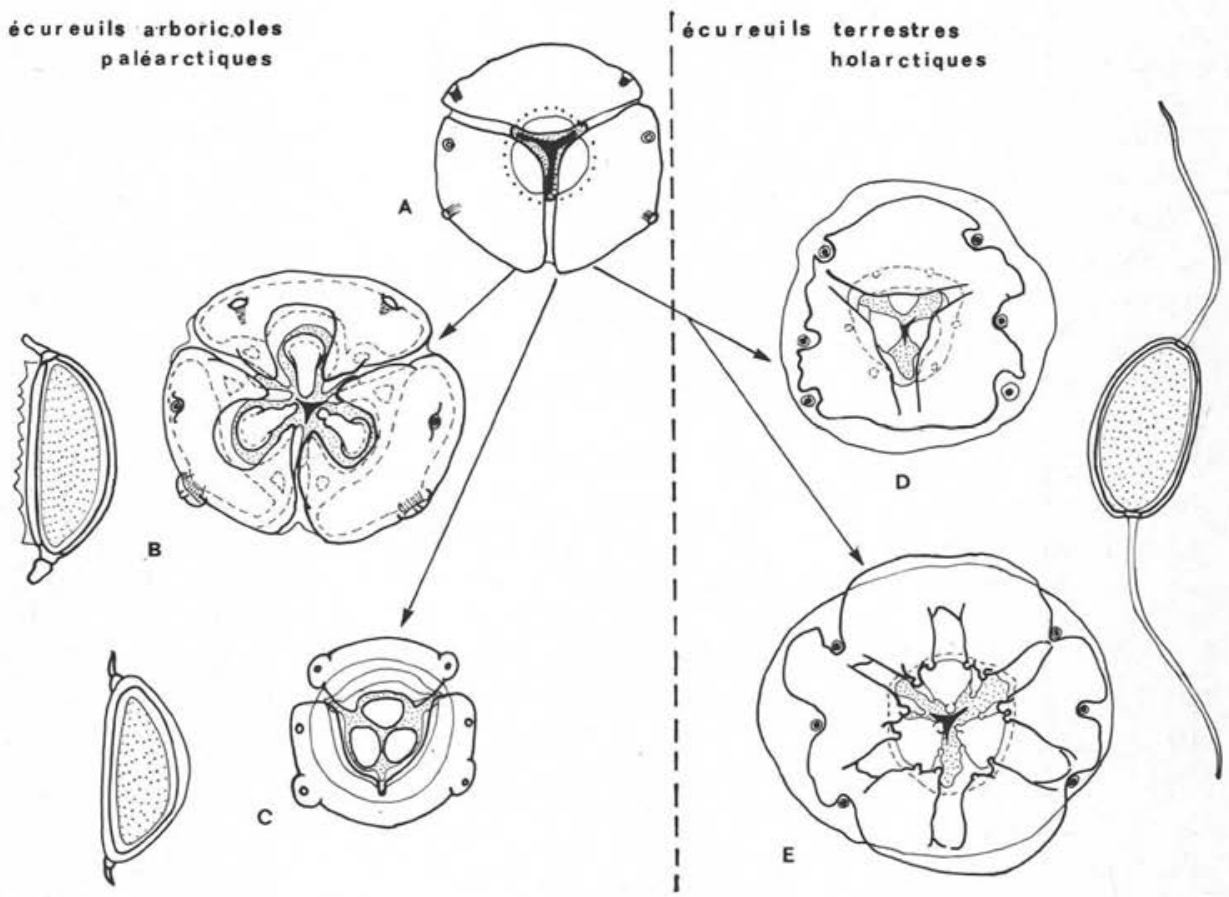

Fig. 4. Arbre phylétique supposé des différents types céphaliques dans le genre Citellina Prendel, 1928. A, C. levini (d'après Li, 1933) ; B, C. petrovi Schulz, 1930 ; C, C. himalensis Inglis et Ogden, 1965 ; D, C. undulata n. sp. ; E, C. caligata (Quentin, 1968).

- groupe I. - Morphologie céphalique primitive de type A, B ou C (lèvres recouvrant le plateau céphalique; terminaisons nerveuses portées par les lèvres). Eufs sans prolongements polaires, ou avec des prolongements courts. Parasites de Sciuridés paléarctiques arboricoles. 
- groupe II. - Morphologie céphalique évoluée de type D ou E (lèvres confluentes ne recouvrant pas le plateau céphalique, qui porte les terminaisons nerveuses). Eufs portant de très longs prolongements polaires. Parasites de Sciuridés holarctiques terrestres.

La parenté du genre Citellina avec le genre Auchenacantha Baylis, 1929, parasite de Dermoptères, a été plusieurs fois soulignée (voir Inglis, 1965 et Quentin, 1973). Nous en trouvons ici deux preuves supplémentaires:

1 - les types céphaliques D et E sont rencontrés dans le genre Citellina chez des hôtes proches : Citellus et Marmota appartiennent, en effet, à la même tribu des Marmotini et ont probablement la même origine néarctique (Moore, 1961). Or, ces 2 types céphaliques sont, dans le genre Auchenacantha, parfois associés dans la même espèce : les mâles étant du type D et les femelles du type E.

2 - l'ornementation cuticulaire décrite plus haut chez $C$. petrovi peut être considérée comme analogue à celle rencontrée chez certaines espèces du genre Auchenacantha: dans les deux genres, en effet, cette ornementation, quand elle existe, est limitée à la partie antérieure du corps, chez les femelles.

Il paraît, en même temps, possible d'éliminer l'hypothèse d'une filiation directe Auchenacantha-Citellina, que l'antériorité des Dermoptères par rapport aux Sciuridés pourrait suggérer. Trois arguments s'opposent à une telle filiation :

- les types céphaliques A et B décrits chez Citellina sont plus archaïques que ceux connus chez Auchenacantha.

- Quentin, Betterton et Krishnasamy (sous presse) ont récemment montré que l'ornementation cuticulaire particulière rencontrée chez certaines femelles est un caractère archaïque : dans le genre Auchenacantha, ce caractère ne persiste qu'à l'état larvaire chez les espèces les plus évoluées.

- Les Oxyures de Sciuridés, dans la région orientale, où ces animaux vivent en contiguité avec les Dermoptères, sont bien connus. Or, non seulement aucun Citellina n'y a été décrit, mais, dans cette région, les Ecureuils sont parasités par des Oxyures appartenant au genre Syphacia Seurat, 1915, ou Sypharista Quentin, 1971, qui ne présentent aucune affinité morphologique avec le genre Auchenacantha.

Il semble donc probable que les genres Auchenacantha et Citellina, quoique très proches, soient séparés depuis longtemps.

Enfin, certains éléments permettent peut-être d'éclairer l'origine géographique du genre Citellina. On peut, en effet, faire les observations suivantes :

- la répartition géographique et le spectre d'hôtes du genre, à une exception près, sont exclusivement holarctiques,

- le type céphalique A, le plus archaïque, est rencontré chez Sciurus, 1'un des plus vieux Ecureuils connus à l'état fossile, en Chine, c'est-à-dire dans une région considérée comme le berceau possible des Sciuridés (Moore, 1961, Lavocat, 1962), 
— cette région est proche de la sous-région Malaise où vivent actuellement les Dermoptères,

- Callosciurus, qui est le seul hôte non strictement holarctique, abrite un parasite dont les structures céphaliques peuvent être considérées comme différenciées. Cet Ecureuil est endémique dans toute la Région Orientale, où il est parasité par des Syphacia (Quentin et Krishnasamy, 1975, Quentin, 1977). La présence d'un Citellina, chez cet hôte, à la frontière des Régions orientales et paléarctique, évoque donc un phénomène de capture. Or, Durette-Desset (1967) a observé, chez d'autres Nématodes, que ce phénomène ne semble se produire qu'à proximité du centre de dispersion de l'espèce primitive et qu'il aboutit, chez les hôtes nouveaux, à des spéciations donnant des formes évoluées,

- Pteromys, qui est strictement paléarctique, est le seul Petauristiné du spectre d'hôtes. Les Petauristinés, y compris le genre Glaucomys, qui est l'équivalent américain de Pteromys, sont habituellement parasités par des Syphacia ou des Sypharista. Des Syphacia ont également été décrits chez Pteromys en Yakutie par Gubanov (1964). Il semble donc qu'il s'agisse ici également d'un phénomène de capture. La morphologie primitive de la tête, qui semble dériver directement du type A observé chez Sciurus, et la présence du parasite dans toute la zone de répartition de son hôte, indiqueraient simplement que cette capture est ancienne.

Ainsi plusieurs arguments semblent converger, qui donnent au genre Citellina une origine centre-est asiatique, d'où il se serait développé chez des Sciuridés anciens. Ces parasites se seraient ensuite différenciés et dispersés avec leurs hôtes dans la région holarctique, atteignant avec les Ecureuils terrestres leur vaste répartition actuelle.

\section{Bibliographie}

Ablassov N.-A. (1962) : Nouvelle espèce de Nématode du groupe Syphacia, Seurat, 1916, de Sciurus vulgaris exalbidus. Izvestia Acad. Sc. Kirgiz, SSR Ser. Biol., 4, 179-181 (en russe).

Akhtar S. A. (1956) : On a new Oxyurid (Nematoda) parasitic in the Kaghan marmot. Proc. Pakistan Acad. Sci., 1, $1-5$.

Baylis H.A. (1929): Some new parasitic Nematodes and Cestodes from Java. Parasitology, 21, 256-265.

Chabaud A.-G., Biocca E. (1955): Vicariances spécifiques (et non génériques) chez des Oxyures parasites de Xerus africanus. Description de Syphacia transafricana n. sp., division du genre Syphacia Seurat, 1916. Bull. Soc. zool. Fr., 80, 124-131.

Chabaud A.-G., Petter A.-J. (1959): Les Nématodes parasites de Lémuriens Malgaches. II. Un nouvel Oxyure: Lemuricola contagiosus. Mem. Inst. scient. Madagascar, sér. A, 13, 127-132.

Durette-Desset M.-C. (1967): Evolution des Nématodes Héligmosomes en rapport avec celle de leurs hôtes fondamentaux, les Microtidae. C.R. Acad. Sci. Paris, 265, 1500-1503.

Dubinina M. N. (1947): The parasitic worms of mammals of Daursk steppe (Abstract). In: Rabot. Akad. Nauk SSSR, Odtel Biol. Nauk, 1945, 178-179 (en russe), (original non vu).

Erhardova-Kotrla B., Daniel M. (1970) : Parasitic Worms of Small Mammals from the Mountain Regions of the Eastern Hindu Kush. Folia Parasit., Praha, 17, 201-216.

Gubanov N.-M. (1964): Helminthofaune des Mammifères d'importance économique dans le Yakut ASSR. Izdaltesvo « Nauka », 1-164 (en russe). 
Hall M.C. (1916) : Nematode parasites of Mammals of the order Rodentia, Lagomorpha and Hyracoidea. Proc. U.S. Natl Mus., 50, 1-258.

Inglis W. G., Ogden C. G. (1965): Observations on the Nematode genus Citellina: with the description of a new species, Citellina himalensis. J. Helminth., 39, 11-18.

Jettmar H. M., Anschau M. (1951) : Beobachtungen and Parasiten steirischer Murmeltiere (Arctomys marmota). Z. Tropenmed. Parasit., 2, 412-428.

Kamiya H., Ishigari K., Yamashita J. (1974): Citellina petrovi Schulz, 1930, from the Japanese flying squirrel, Pteromys volans orii Kuroda. Jap. J. Vet. Res., 22, 116-120.

Korneev (1951) : In : Principales of Nematodology. VIII. Oxyurata of Animals and Man, Moscow. Acad. Sci. USSR, 557 p. (en russe).

Lavocat R. (1962) : Réflexions sur l'origine et la structure du groupe des Rongeurs. Editions du CNRS, Paris. Colloques internationaux du CNRS, $\mathrm{n}^{\circ}$ 104, 287-299.

Li H. C. (1933) : Report on a collection of parasitic Nematodes, mainly from China. III. Oxyuroidea. Chin. Med. J., 47, 1307-1325.

Manter H.W. (1930): Two new nematodes from the woodchuck, "Marmota monax canadensis». Trans. Am. Microsc. Soc., 49, 26-33.

Merkuscheva I. V. (1955): A new nematode Citellina skrjabini nov. sp. from the intestine of a spotted Souslik (Citellus suslica Güld) in White Russian SSR. Izvest. Akad. Nauk. Belorussk. SSR (4), 103-106 (en russe).

Moore J.C. (1961): The Spread of Existing Diurnal Squirrels across the Bering and Panamanian Land Bridges. Am. Mus. Navitates, $\mathrm{n}^{\circ}$ 2044, 1-26.

Petrov A. M., Bayanov M. G. (1962) : Syphacia (Syphatineria) toshevi, sp.n., nouveau Nématode de l'intestin de l'Ecureuil. Zool. J. Acad. Sci., U.R.S.S., 41, 1102-1105 (en russe).

Prendel A.R. (1928) : Zur Kenntnis der Darm-helminthen einiger Nagetiere. Rev. Microbiol. Epidem. Parasitol., 7, 410-416.

Quentin J.-C. (1968): Description de Citellina sp. Oxyure de Marmotte d'Amérique du Nord. Bull. Mus. Nat. Hist. Nat., $2^{\circ}$ sér., 40, 210-213.

Quentin J.-C. (1971) : Description d'un nouvel Oxyurinae: Sypharista kamegaii n. gen., n. sp., parasite d'un Ecureuil volant du Japon. Bull. Mus. Nat. Hist. Nat., $2^{e}$ sér., 42, 989-995.

Quentin J.C. (1973): Les Oxyurinae de Rongeurs. Bull. Mus. Nat. Hist. Nat., 3e sér., Zool. 112, 1045-1096.

- (1977): Sur la morphologie de Syphacia callosciuri n.sp., Oxyure parasite d'un Ecureuil de Malaisie, et sur l'origine de l'Oxyure du Rattus: S. muris (Yamaguti, 1935). Ann. Parasitol. Hum. Comp., 52, 559-567.

Quentin J.-C., Betterton C., Krishnasamy M.: Oxyures nouveaux ou peu connus, parasites de Primates, de Rongeurs et de Dermoptères en Malaisie. Création du sous-genre Coloenterobius n. subgen. Bull. Mus. Nat. Hist. Nat. (sous presse).

Quentin J.-C., Krishnasamy M. (1975): Oxyures de Rongeurs. I. Spéciations des Oxyures parasites de Rongeurs Petauristinés en Malaisie. Mém. Mus. Nat. Hist. Nat., Paris, Nelle sér. Ser. A, Zool., 94, 1-49.

Quentin J.-C., Ténora F. (1974): Morphologie et position systématique de Lemuricola (Rodentoxyuris) sciuri (Cameroun, 1931) nov. comb., nov. subgen., et Syphacia (Syphatineria) funambuli Johnson, 1967, Oxyures (Nematoda) parasites de Rongeurs Sciuridés. Bull. Mus. Nat. Hist. Nat., Paris, 3e sér., Zool., 178, 1525-1536.

Rausch R., Tiner J.D. (1948) : Studies on the Parasitic Helminths of the North Central States. I. Helminths of Sciuridae. Am. Midl. Nat., 39. 728-747.

Read C. P. (1957) : The Oxyurid nematodes of rodents. I. The genus Citellina. J. Parasit., 43, 446-449.

Schulz R.E.S. (1930): (The Helminth Parasites of Rodents in the Severo-Dvinsk Region). In : Results of the 32nd and 29th Helminth. Expedition, 1926-1927 (en russe).

Seurat L.-G. (1915) : Sur deux nouveaux Oxyures du Maroc. Bull. Soc. Hist. Nat. Afr. N., 7, 24-31.

Spassky A. A., Rijikov K. M., Sudarikov V.E. (1950) : Notes on the helminth fauna of the Menzbier marmot (Marmota menzbieri Kashk.). Trud. gel'mint. Lab. AN. SSSR, 4, $32-39$ (en russe). 